Sharif University of Technology
Scientia Iranica
Transactions E: Industrial Engineering
SCIENTIA
I RAN I C A

\title{
Sustainable supply chain design with water environmental impacts and justice-oriented employment considerations: A case study in textile industry
}

\author{
H.R. Jafari ${ }^{a}$, M. Seifbarghy ${ }^{\mathrm{b}, *}$ and M. Omidvari ${ }^{\mathrm{c}}$ \\ a. Department of Industrial Engineering, Science and Research Branch, Islamic Azad University, Tehran, Iran. \\ b. Department of Industrial Engineering, Alzahra University, Tehran, Iran. \\ c. Facility of Industrial and Mechanical Engineering, Qazvin Branch, Islamic Azad University, Qazvin, Iran.
}

Received 13 December 2015; received in revised form 16 April 2016; accepted 30 May 2016

\section{KEYWORDS}

Sustainable supply chain network design; Justice-oriented employment; Water consumption; Textile industry.

\begin{abstract}
The concept of sustainability in supply chain management refers to a logical balance between economic development, environmental considerations, and social responsibilities. In this paper, a sustainable model has been proposed to design a supply chain network in textile industries considering the key environmental and social factors. Regarding the type of industry and characteristics of the area under study (Zanjan, northwest Iran), minimizing the negative effects of wasteful extraction of ground waters and the environmental pollution resulting from industrial wastewaters and maximizing justicebased employment were considered. The supply chain consists of the following elements: suppliers, plants, distribution centers, water refinery centers, and customer zones. One of the important features of the proposed model is that it considers the lost opportunity cost of facilities and focuses on wastewater recycling in water refineries. To solve the model, the Multi-Objective Vibration Damping Optimization (MOVDO) algorithm has been used. In addition, to evaluate the proposed model, as a case study, the supply chain network design problem was solved in textile industry. In addition, to evaluate the solution performance of the used algorithm in comparison with that of the NSGA-II algorithm, ten random problems with different sizes were solved, and the results were analyzed using different indexes. All in all, the results show that the proposed method has the necessary performance.
\end{abstract}

(C) 2017 Sharif University of Technology. All rights reserved.

\section{Introduction}

A supply chain consists of multiple members, such as suppliers, plants, Distribution Centers (DCs), and customers, in which suppliers provide raw material for plants, and the plants manufacture products based on customers' requirements (demands) and transport them through channels to DCs which are responsible

\footnotetext{
*. Corresponding author.

E-mail addresses: h.r.jafari2015@gmail.com (H.R. Jafari); seifbar@yahoo.com(M.Seifbarghy);omidvari88@yahoo.com (M. Omidvari)
}

for the distribution of final products to the costumer's zones $[1,2]$.

A cursory look at SCM literature reveals that it consists of two major parts: Forward Logistic (FL) and Reverse Logistic (RL). The combination of these two parts, called closed-loop supply chain, includes more elements such as collection centers, recycle centers, and disposal centers that generally serve the objective of minimization of waste materials and products. In addition, state laws force manufacturers to focus on End Of Life (EOL) of their products and also assume the social and environmental responsibilities of their business [3]. Furthermore, reverse supply chain has 
important features such as preventing the waste of resources, reducing environmental pollution, increasing the profitability of second-handed goods, promoting competitiveness, and increasing the efficiency of the supply chain. Thanks to the emergence of the concept of sustainability, the field of SCM has developed substantially. In fact, sustainability seeks to strike a logical and appropriate balance between economic development, environmental considerations, and social responsibilities. Therefore, the main goal of a sustainable supply chain is to integrate the three dimensions of a sustainable development, including economic, social, and environmental goals, resulting from requirements of customers and stakeholders [4].

In this paper, we proposed a multi-objective, multi-echelon, and multi-product supply chain design problem in which some plants produce a number of products assembled from specific parts supplied by a number of suppliers. The finished products go through certain DCs and are transported to costumers' zones using some existing transportation channels. In addition, designing a sustainable closed-loop supply chain concentrating on water recycling is one of the main features of the presented problem. Most research studies tend to focus on product recycling, while this paper formulates by-product material recycling in production processes (e.g., water, oil, and so forth). The findings of this research are of utmost importance in areas encountering water shortage crisis. In particular, textile, paper, and steel manufacturing industries have been always struggling with numerous challenges resulting from the lack of water. We have worked on a real-life case in textile industry whose supply chain is located in Zanjan (in North-West of Iran); Paretobased approaches are also used in solving the problem.

The remainder of this article is structured as follows: in Section 2, the literature review is presented. In Section 3, the problem at hand is formulated. In Section 4 , the solution method for this model is determined. Section 5 provides a case study, and Section 6 analyzes the results and comparisons. Finally, the conclusion and future works are given in Section 7 .

\section{Literature review}

Forward supply chain consists of a series of activities which result in the conversion of raw materials to finished products. Therefore, managers attempt to improve supply chain performances by considering the features of reverse supply chain such as the facilities of collection, recovery, and disposal centers with reverse flow in a logistic network. It is also important to determine their locations and capacities, buffer inventories, and product flows between the facilities in a supply chain network. Hassanzadeh-Amin and Zhang [5] proposed a fuzzy mathematical programming model for a closed-loop supply chain which considers multiple depots, multiple vehicles, multiple products, multiple customers, and different time periods. In their research, demand was considered to be fuzzy. Zhang et al. [6] presented a closed-loop supply chain network consisting of multiple plants, collection centers, demand markets, and products. Therefore, a mixedinteger linear programming model was proposed that minimizes the total cost. They investigated the impact of demand and return uncertainties on the network configuration by stochastic programming.

In the supply chain environment, the environmental and social consequences of business systems have received great deal of attention in recent years. During these years, minimizing the total cost or maximizing the profit has been the main objective of supply chains. Nowadays, businesses are considered to be responsible for the environmental impacts of their products and operations, the health and safety of their employees in particular and the whole society in general. In sustainable supply chains, Tang and Zhou [7] reviewed the recent developments of operational research/management science in the domain of environmentally and socially sustainable operations. Brandenburg et al. [8] studied the quantitative models that address sustainability aspects in a forward supply chain. For decades, many papers have focused on SCND due to the increasing importance of sustainability, while some other articles have considered environmental and/or social impacts of SCND as additional objective(s) of multi-objective SCND. For more details about sustainability research studies, one can refer to Wang et al. [9], Elhedhli and Merrick [10], Chen et al. [11], and Govindan et al. [12]. Kannan et al. [13] filled the gap between SCND and the Order-Allocation Problem (OAP) through sustainable OAP and SCND. Their multi-objective optimization model is a fiveechelon supply chain network. To solve the model, two multi-objective algorithms were applied based on electromagnetism-like mechanism and variable neighborhood search.

In the related research studies, many techniques have been used to solve supply chain design problems. One of the main approaches is mathematical programming in which the problem is formulated as a Mixed Integer Programming (MIP) model. Cakravastia et al. [14] used a MIP model for supplier selection in a multi-stage supply chain design problem. One of the most important advantages of using MIP model is its ability in finding the exact optimal solution. But, as a disadvantage of these approaches, one can refer to the amount of computation time that increases as the problem size increases. Another technique that is applied to the solving process of the supply chain design problem is the heuristic approach. In other words, since the multi-stage design problem is difficult to solve, 
Santoso et al. [15] used heuristic approaches to solve this problem. Syam [16] applied a Simulated Annealing algorithm (SA) for a multi-source, multi-product, and multi-location framework. Syarif et al. [17] developed a spanning tree-based Genetic Algorithm (GA) approach for the SCN design problem. Likewise, Jayaraman and Ross [18] proposed a simulated annealing for the design of distribution network. Yeh [19] presented a greedy method, a linear programming technique, and three local search methods to solve the supply chain design problem. Altiparmak et al. [20] presented a GAbased procedure to design a four-echelon supply chain including suppliers, plants, warehouses, and customers. Yeh [21] proposed a Memetic Algorithm (MA), which is a combination of GA, greedy heuristic, and local search methods, for the same problem. Moncayo-Martinez and Zhang [22] proposed an algorithm based on Pareto Ant Colony Optimization as an effective meta-heuristic method to solve multi-objective supply chain design problems. As an advantage, although the heuristic techniques do not necessarily find the optimum solutions, they can produce a near-optimum solution with a rational computation time. In their paper, multiobjective version of Vibration Damping Optimization (VDO) was presented to solve the model. VDO, presented by Mehdizadeh and Tavakkoli-Moghaddam [23], is based on the concept of vibration damping in mechanical vibration. Hajipour et al. [24] introduced a multi-objective version of VDO for optimization problems. In their paper, to solve the proposed supply chain design problem, a multi-objective version of VDO was applied. Finally, to learn more about the applications of VDO and MOVDO algorithms, one can refer to [25-28] in which these algorithms have been used to solve the queuing-location-allocation, lotsizing, location-pricing, and facility location-allocation problems. Therefore, as the main contribution of the present study, a multi-objective, multi-echelon, and multi-product supply chain network design model with sustainable considerations is presented to optimize the total cost of supply chain and reduce the potential negative environmental and social effects simultaneously. Moreover, an efficient Pareto-based algorithm based on vibration damping is presented to solve the proposed model in supply chain design scope.

\section{Model description}

In this section, we first define the problem at hand; subsequently, the model formulation is elaborated in detail. The proposed mathematical model is a singleperiod strategic supply chain network design with multiple suppliers, multiple manufacturing plants, a set of candidate DCs, and costumers in the network. The main users of the model are policy-making organizations; for example, government as the major policy- maker, with the objective of designing a sustainable supply chain, can always use the proposed model in its strategic decisions. In fact, the proposed model provides a sustainable supply chain network design in a long-term period considering the recycling policy of critical materials such as water. Textile and steel industries with a high volume of water consumption are the major users of the given model. In this context, the main contributions of this paper are summarized as follows:

- Designing a sustainable closed-loop supply chain concentrating on water recycling as a consuming byproduct material in most industries. Most research studies focus on product recycling, while in this paper, the emphasis is on the recycling of by-product materials in production processes (e.g. water, oil, and so forth). The findings of the present research are of utmost importance for the areas encountering water shortage crisis;

- Formulating the sustainable supply chain problem at hand with an approach based on clustering of location areas in terms of environmental and social factors.

Considering the concept of sustainability, the goals of this study are of three dimensions:

(I) Economic dimension: Minimizing the total costs of system including fixed cost of establishing facilities, transportation costs of material flows, production costs, and lost opportunity cost of facilities (including plants, DCs, and Water Refinery Centers (WRCs));

(II) Environmental dimension: Minimizing the total water consumption from ground waters considering the negative effects of water extraction in each water environmental cluster along with industrial wastewater (e.g., these two factors are highlighted in textile industries);

(III) Social dimension: Maximizing the total justice-oriented employment with regard to the priorities in different social clusters.

To provide an elaborate description, the framework of the proposed mathematical model is presented in Figure 1.

In order to explain the problem, the assumptions are given as follows:

- Since the model is designed in strategic level for a long-term period, it is a single-period decisionmaking problem;

- Potential plants and DCs have constant capacities;

- Multiple products are considered; 


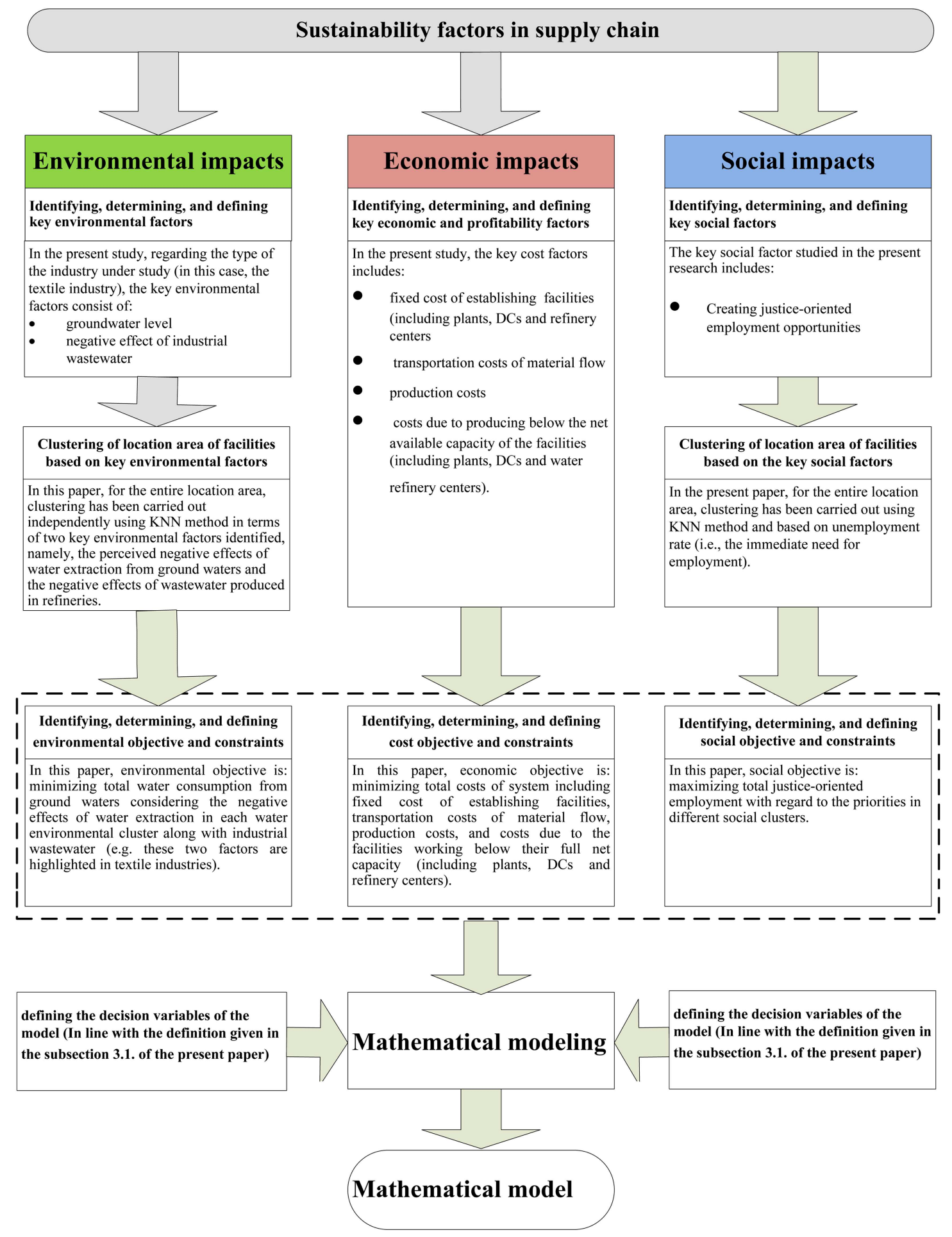

Figure 1. The mathematical modeling framework.

- The entire area under study is clustered based on key social and environmental factors separately;

- There is a lower bound for the minimal employment required in each region.

- There is an upper bound for disposal (wastewater) in each region;
- There are multiple transportation channels between facilities;

- Potential locations of facilities (including plants, DCs, and WRCs) are in discrete locations;

- Each product is assembled in the plants through multiple parts supplied from different suppliers; 
- Produced products in the plants are transported to the customers through DCs;

- The transportation costs and times are different between facilities;

- The establishment of plants, DCs, and refinery centers has social and environmental effects.

\subsection{Notations}

The following notations are used to state the mathematical model:

Sets:

$S \quad$ Set of raw materials suppliers ( $s$ : index of suppliers)

$U$ Set of potential water resources $(u$ : index of water resources)

$I \quad$ Set of potential plants ( $i$ : index of plants)

$J \quad$ Set of potential DCs ( $j$ : index of DCs)

$K \quad$ Set of costumers ( $k$ : index of costumers)

$R \quad$ Set of potential Water Refinery Centers (WRCs) ( $r$ : index of WRCs)

$P \quad$ Set of products ( $p$ : index of products)

$M \quad$ Set of raw materials ( $m$ : index of raw materials)

$Z \quad$ Set of social zones clustered based on employment rate criterion ( $z$ : index of social clusters)

$W \quad$ Set of environmental zones $1(w$ : index of environmental cluster 1 clustered based on groundwater level)

$H \quad$ Set of environmental zones $2(h$ : index of environmental cluster 2 clustered based on negative effects of industrial wastewater)

$C_{z} \quad$ Set of candidate facilities to locate in social cluster $z$

$D_{w} \quad$ Set of candidate facilities to locate in environmental $w$ cluster

$L_{h} \quad$ Set of candidate facilities to locate in social cluster $h$

$L S_{s i} \quad$ Set of transportation channels $(l)$ between nodes $s$ and $i$

$L U_{u i} \quad$ Set of transportation channels $(l)$ between nodes $u$ and $i$

$L P_{i j} \quad$ Set of transportation channels $(l)$ between nodes $i$ and $j$

$L O_{i r} \quad$ Set of transportation channels $(l)$ between nodes $i$ and $r$

$L R_{r i} \quad$ Set of transportation channels $(l)$ between nodes $r$ and $i$
$L D_{j k} \quad$ Set of transportation channels $(l)$ between nodes $j$ and $k$

Parameters:

$c s_{\text {siml }} \quad$ Unit transportation cost of raw material $m$ from supplier $s$ to plant $i$ using transportation channel $l$

$c p_{i j p l} \quad$ Unit transportation cost of product $p$ from plant $i$ to $\mathrm{DC} j$ using transportation channel $l$

$c d_{j k p l} \quad$ Unit transportation cost of product $p$ from DC $j$ to costumer $k$ using transportation channel $l$

$\mathrm{CO}_{\text {irl }} \quad$ Unit transportation cost of wastewater from plant $i$ to water refinery center $r$ using transportation channel $l$

$c r_{\text {ril }} \quad$ Unit transportation cost of purified water from WRC $r$ to plant $i$ using transportation channel $l$

$M S_{s m} \quad$ Capacity of supplier $s$ for raw material $m$

$M U_{u} \quad$ Capacity of water resource $u$ (in liters)

$M P_{i} \quad$ Capacity of plant $i$ (net available time)

$M R_{r} \quad$ Capacity of WRC $r$ for water refinery (in liters)

$M D_{j} \quad$ Capacity of DC $j$ (in product unit)

$F P_{i} \quad$ Fixed cost to open plant $i$

$F R_{r} \quad$ Fixed cost to open recycling center $r$

$F D_{j} \quad$ Fixed cost to open DC $j$

$D_{k p} \quad$ Demand for product $p$ in costumer's zone $k$

$p c_{i p} \quad$ Unit production cost for product $p$ at plant $i$

$r c_{r} \quad$ Unit water refinery cost at WRC $r$

$\rho_{i} \quad$ Percentage of produced recyclable wastewater in plant $i$

$\lambda \quad$ Percentage of water loss at WRCs (after refinery process)

$\gamma_{i} \quad$ The cost of lost opportunity (below full-capacity operation) in plant $i$ $(\$ / \min )$

$\varepsilon_{r} \quad$ The cost of lost opportunity (below full-capacity operation) in WRC $r$ $(\$ / \min )$

$\tau_{j} \quad$ The cost of lost opportunity (below full-capacity operation) in DC $j$

$\eta_{h} \quad$ Environmental coefficient of industrial wastewater's negative effects compared to other environmental factors $0 \leq \eta_{h} \leq 1$ 
$\eta_{w}$

Environmental coefficient of water extraction from groundwater (negative effects) compared to other environmental factors $0 \leq \eta_{w} \leq 1$ and $\eta_{h}+\eta_{w}=1$

$\psi_{w} \quad$ Weighted coefficient of negative effects of water extraction from groundwater resources on environmental cluster $w$

$e_{i} \quad$ Number of employment opportunities provided in plant $i$

$n_{j} \quad$ Number of employment opportunities provided in DC $j$

$h_{r} \quad$ Number of employment opportunities provided in refinery center $r$

$w_{z} \quad$ Priority of creating employment opportunities in social cluster $z$

$M S_{z} \quad$ The least necessary number of employment opportunities in social cluster $z$ based on governmental policies

$M F_{h} \quad$ An upper bound of industrial wastewater produced by refineries in environmental cluster $h$ (based on governmental policies)

$M W_{w} \quad$ An upper bound of water extraction from groundwater in environmental cluster $w$

$a_{m p} \quad$ Consumption rate of raw material $m$ for product $p$

$b_{p} \quad$ Consumption rate of water for product $p$

$v_{i p} \quad$ Cycle time of product $p$ in plant $i$ (in minutes)

Decision variables:

$P L_{i} \quad$ A binary variable which is 1 if plant $i$ is opened, and 0 otherwise

$\mathrm{DC}_{j} \quad$ A binary variable which is 1 if $\mathrm{DC} j$ is opened, and 0 otherwise

$R C_{r} \quad$ A binary variable which is 1 if WRC $r$ is opened, and 0 otherwise

$X_{\text {siml }} \quad$ A binary variable which is 1 if transportation channel $l$ between supplier $s$ and plant $i$ is used for shipping raw material $m$, and 0 otherwise

$Y_{i j p l} \quad$ A binary variable which is 1 if transportation channel $l$ between plant $i$ and DC $j$ is used for shipping product $p$, and 0 otherwise

$Z_{j k p l} \quad$ A binary variable which is 1 if transportation channel $l$ between DC $j$ and customer $k$ is used for shipping product $p$, and 0 otherwise
$T_{\text {uil }} \quad$ A binary variable which is 1 if transportation channel $l$ between supplier $u$ and plant $i$ is used for shipping water, and 0 otherwise

$V_{i r l} \quad$ A binary variable which is 1 if transportation channel $l$ between plant $i$ and WRC $r$ is used for shipping recyclable water, and 0 otherwise

$W_{\text {ril }} \quad$ A binary variable which is 1 if transportation channel $l$ between WRC $r$ and plant $i$ is used for shipping recycled water, and 0 otherwise

$Q S_{\text {siml }} \quad$ Quantity of raw material $m$ transported from supplier $s$ to plant $i$ using transportation channel $l$

$Q U_{\text {uil }} \quad$ Quantity of water transported from water resource $u$ to plant $i$ using transportation channel $l$

$Q_{i p} \quad$ Quantity of product $p$ produced at plant $i$

$Q P_{i j p l} \quad$ Quantity of product $p$ transported from plant $i$ to DC $j$ using channel $l$

$Q D_{j k p l} \quad$ Quantity of product $p$ transported from DC $j$ to customer $k$ using channel $l$

$Q O_{i r l} \quad$ Quantity of water transported from plant $i$ to WRC $r$ using transportation channel $l$

$Q R_{\text {ril }} \quad$ Quantity of water transported from WRC $r$ to plant $i$ using transportation channel $l$

In order to provide an elaborate description of the model structure, Figure 2 illustrates the configuration of the supply chain under study.

\subsection{Model formulation}

The problem can be formulated as follows:

$$
\begin{aligned}
\min f_{1} & =\sum_{i} F P_{i} \cdot P L_{i}+\sum_{j} F D_{j} \cdot \mathrm{DC}_{j} \\
& +\sum_{r} F R_{r} \cdot R C_{r}+\sum_{i} \sum_{p} p c_{i p} \cdot Q_{i p} \\
& +\sum_{i} \sum_{r} \sum_{l \in L O_{i r}} r c_{r} \cdot Q O_{i r l} \\
& +\sum_{s} \sum_{i} \sum_{m} \sum_{l \in L S_{s i}} c s_{s i m l} \cdot Q S_{s i m l} \\
& +\sum_{u} \sum_{i} \sum_{l \in L U_{u i}} c u_{u i l} \cdot Q U_{u i l} \\
& +\sum_{i} \sum_{j} \sum_{p} \sum_{l \in L P_{i j}} c p_{i j p l} \cdot Q P_{i j p l}
\end{aligned}
$$




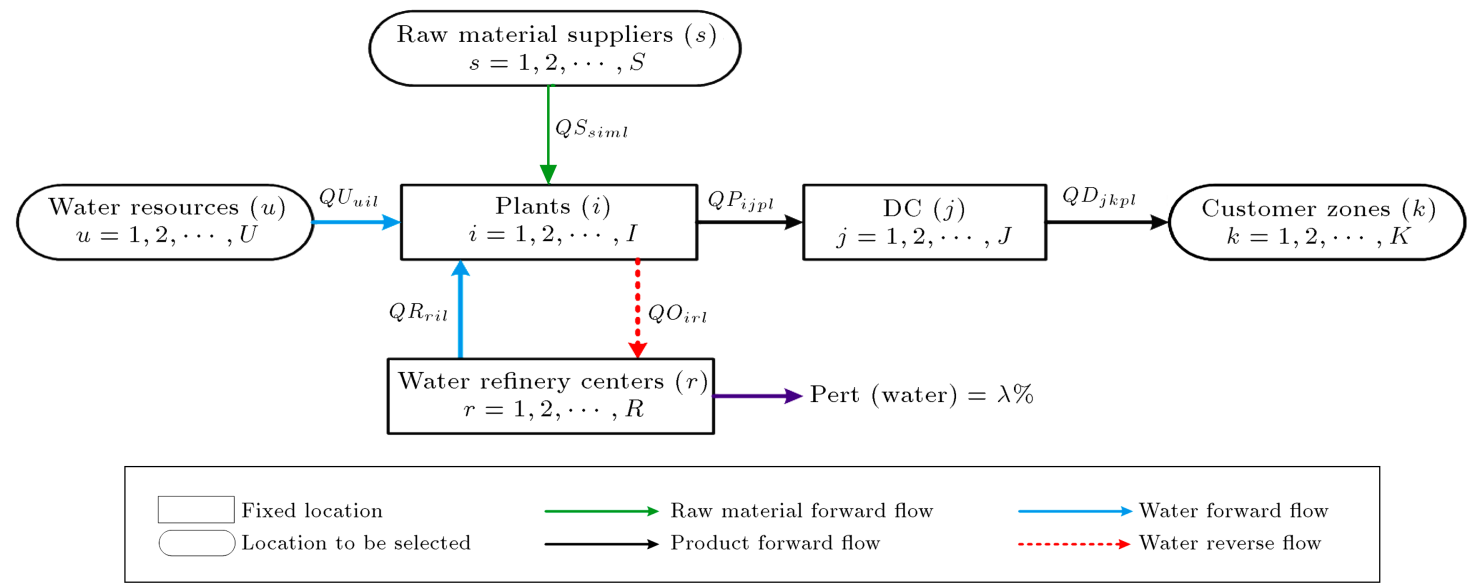

Figure 2. Graphical configuration of the supply chain.

$$
\begin{aligned}
& +\sum_{j} \sum_{k} \sum_{p} \sum_{l \in L D_{j k}} c d_{j k p l} \cdot Q D_{j k p l} \\
& +\sum_{i} \sum_{r} \sum_{l \in L O_{i r}} c O_{i r l} . Q O_{i r l} \\
& +\sum_{r} \sum_{i} \sum_{l \in L R_{r i}} c r_{r i l} \cdot Q R_{r i l} \\
& +\sum_{i} \gamma_{i} \cdot P L_{i} \cdot\left(M P_{i}-\sum_{p} v_{i p} Q_{i p}\right) \\
& +\sum_{r} \varepsilon_{r} \cdot R C_{r} \cdot\left(M R_{r}-\sum_{i} \sum_{l \in L O_{i r}} Q O_{i r l}\right) \\
& +\sum_{j} \tau_{j} \cdot \mathrm{DC}_{j} \cdot\left(M D_{j}-\sum_{k} \sum_{p} \sum_{l \in L D_{j k}} Q D_{j k p l}\right) \\
& \min f_{2}=\eta_{h} \sum_{h}\left(\sum_{i} \sum_{r \in L_{h}} \sum_{l \in L O_{i r}} Q O_{i r l}\right. \\
& \left.-\sum_{r \in L_{h}} \sum_{i} \sum_{l \in L R_{i r}} Q R_{r i l}\right) \\
& +\eta_{w} \sum_{w}\left(\psi_{w} \sum_{u \in D_{w}} \sum_{i} \sum_{l \in L U_{u i}} Q U_{u i l}\right), \\
& \max f_{3}=\sum_{z} w_{z}\left(\sum_{i \in C_{z}} e_{i} P L_{i}+\sum_{j \in C_{z}} n_{j} \mathrm{DC}_{j}\right. \\
& \left.\left.+\sum_{r \in C_{z}} h_{r} R C_{r}\right)\right)
\end{aligned}
$$

s.t.:

$\begin{array}{ll}\sum_{j} \sum_{l \in L D_{j k}} Q D_{j k p l}=D_{k p} & \forall k, p, \\ \sum_{j} \sum_{l \in L P_{i j}} Q P_{i j p l}=Q_{i p} & \forall i, p, \\ \sum_{k} \sum_{l \in L D_{j k}} Q D_{j k p l}=\sum_{i} \sum_{l \in L P_{i j}} Q P_{i j p l} & \forall j, p,\end{array}$

$\sum_{p} b_{p} \cdot Q_{i p}=\left(\frac{1}{1-\left(\rho_{i}\right)(1-\lambda)}\right) \sum_{u} \sum_{l \in L U_{u i}} Q U_{u i l} \quad \forall i$

$\sum_{p} b_{p} \cdot Q_{i p}-\sum_{u} \sum_{l \in L U_{u i}} Q U_{u i l}=\sum_{r} \sum_{l \in L U_{u i}} Q R_{r i l} \quad \forall i$

$\sum_{l \in L O_{i r}} Q O_{i r l}(1-\lambda)=\sum_{l \in L R_{r i}} Q R_{r i l} \quad \forall i, r$

$\sum_{p} a_{m p} Q_{i p} \leq \sum_{s} \sum_{l \in L S_{s i}} Q S_{\text {siml }} \quad \forall i, m$

$\sum_{i} \sum_{l \in L S_{s i}} Q S_{s i m l} \leq M S_{s m} \quad \forall s, m$

$\sum_{i} \sum_{l \in L O_{i r}} Q O_{i r l} \leq M R_{r} \cdot R C_{r} \quad \forall r$

$\sum_{p} v_{i p} \cdot Q_{i p} \leq M P_{i} \cdot P L_{i}$

$\forall i$

$\sum_{k} \sum_{p} \sum_{l \in L D_{j k}} Q D_{j k p l} \leq M D_{j} \cdot \mathrm{DC}_{j} \quad \forall j$

$\sum_{u \in D_{w}} \sum_{i} \sum_{l \in L U_{u i}} Q U_{u i l} \leq M W_{w} \quad \forall w$ 


$$
\begin{aligned}
& \sum_{i \in C_{z}} e_{i} \cdot P L_{i}+\sum_{j \in C_{z}} n_{j} \cdot \mathrm{DC}_{j}+\sum_{r \in C_{z}} h_{r} \cdot R C_{r} \geq M S_{z} \quad \forall z
\end{aligned}
$$

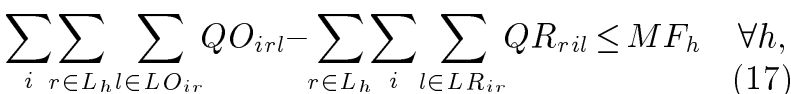

$$
\begin{aligned}
& P L_{i}, \mathrm{DC}_{j}, R C_{r}, X_{s i m l}, Y_{i j p l}, Z_{j k p l}, V_{i r l}, \text { and } \\
& W_{\text {ril }}=\{0,1\} \text {, } \\
& Q_{i p}, Q S_{s i m l}, Q P_{i j p l}, Q D_{j k p l}, Q R_{i r l}, \text { and } \\
& Q O_{r i l} \geq 0 \quad \forall s, i, j, l, k, m, r, p .
\end{aligned}
$$$$
\sum_{s} \sum_{l \in L S_{s i}} X_{s i m l} \leq 1 \quad \forall i, m
$$$$
\sum_{u} \sum_{l \in L U_{u i}} T_{u i l} \leq 1
$$$$
\sum_{i} \sum_{l \in L P_{i j}} Y_{i j p l} \leq 1
$$$$
\sum_{j} \sum_{l \in L D_{j k}} Z_{j k p l} \leq 1
$$$$
\sum_{i} \sum_{l \in L O_{i r}} V_{i r l} \leq 1
$$$$
\sum_{r} \sum_{l \in L R_{i r}} W_{r i l} \leq 1 \quad \forall i
$$$$
\sum_{p} \sum_{l \in L P_{i j}} Y_{i j p l}=\mathrm{DC}_{j} \quad \forall i, j,
$$$$
\sum_{m} \sum_{l \in L S_{s i}} X_{s i m l}=P L_{i} \quad \forall s, i
$$$$
\sum_{l \in L O_{i r}} V_{i r l}=R C_{r} \quad \forall i, r
$$$$
\sum_{l \in L R_{r i}} W_{r i l}=P L_{i} \quad \forall i, r
$$$$
M S_{s m} \cdot X_{s i m l}-Q S_{s i m l} \geq 0 \quad \forall s, i, m, l,
$$$$
M U_{u} \cdot T_{u i l}-Q U_{u i l} \geq 0 \quad \forall u, i, l,
$$$$
M P_{i} . Y_{i j p l}-Q P_{i j p l} \geq 0 \quad \forall i, j, p, l,
$$$$
M D_{j} . Z_{j k p l}-Q D_{j k p l} \geq 0 \quad \forall j, k, p, l,
$$$$
\left(\sum_{j} \sum_{p} \sum_{l \in L P_{i j}} Q P_{i j p l} . b_{p} \cdot \rho_{i} \cdot V_{i r l}\right)-Q O_{i r l} \geq 0
$$$$
\forall i, r, l \text {, }
$$$$
M R_{r} \cdot W_{\text {ril }}-Q R_{\text {ril }} \geq 0 \quad \forall i, r, l,
$$

More descriptions of the above formulations are presented as follows:

- Objective function (1) minimizes the total cost of the system including the fixed cost of establishing facilities (such as plants, DCs, and refinery centers), the transportation costs of material flow, production costs, and the lost opportunity cost of facilities (including plants, DCs, and WRCs);

- Objective function (2) minimizes the total consuming water from ground waters with regard to the negative effects of water extraction on each water-environmental cluster along with industrial wastewater (e.g., these two factors are highlighted in textile industries)

- Objective function (3) maximizes the number of employment opportunities provided in plants, DCs, and WRCs in different social clusters based on its priorities;

- Constraint (4) makes a balance between transported products from DCs to customers with demand customers for the products. According to this restriction, shortage is not allowed; then, all demands must be satisfied;

- Constraint (5) ensures that all produced products are transported into DCs;

- Constraint (6) makes a balance between transported products from plants into DC with transported products from DC to costumers;

- Constraint (7) makes a balance between input quantities of recyclable materials and plants with their outputs;

- Constraint (8) makes a balance between required water in a plant with extracted water from groundwater;

- Constraint (9) makes a balance between input quantities of recyclable materials to plant with their outputs with regard to reverse cycle of recycling materials;

- Constraint (10) makes a balance between transported materials from suppliers to plants with produced products with regard to the consumption coefficient of material in end-products;

- Constraint (11) assigns an upper bound to the capacities of suppliers in order to supply material; 
- Constraint (12) assigns an upper bound to the capacities of refineries in water refinery;

- Constraint (13) assigns an upper bound to the capacities of the plants;

- Constraint (14) assigns an upper bound to the capacities of the DCs (for distributor);

- Constraint (15) assigns an upper bound to the water extraction from different groundwater capacities in each environmental cluster;

- Constraint (16) assigns a lower bound to the required hiring occupation in each social cluster;

- Constraint (17) assigns an upper bound to the wastewater produced by refineries in each environmental cluster;

- Constraints (18)-(23) ensure that each facility supplies its requirement through only one supplier in the previous echelon;

- Constraints (24)-(27) ensure that DCs, plants, and RCs are not opened if they have no active incident transportation channel on it;

- Constraints (28)-(33) indicate that the flow can be done only through active transportation channels;

- Constraints (34) give the status of the decision variables.

\section{A Pareto-based approach}

Due to the complexity of the supply chain design problems, metaheuristic algorithms can be used to solve them. In the literature, the lack of a good algorithm which leads to a near-optimum solution in a rational CPU time to solve the supply chain design problem is still felt $[2,6]$. With regard to the complexity of the proposed supply chain design problem, in this section, a Pareto-based meta-heuristic algorithm, called MOVDO, is proposed to solve the proposed multiobjective mathematical formulation at hand. However, some required multi-objective backgrounds are first defined in the following subsection.

\subsection{Solution representation}

To code the solutions, we presented a five-fold solution representation structure (Figure 3 ) in the form of the following descriptions:

\begin{tabular}{r|c|c|c|c|}
\cline { 2 - 5 } Part (I) & 1 & 2 & $\cdots$ & $S$ \\
\cline { 2 - 5 } Part (II) & 1 & 2 & $\cdots$ & $U$ \\
\cline { 2 - 5 } Part (III) & 1 & 2 & $\cdots$ & $I$ \\
\cline { 2 - 5 } Part (IV) & 1 & 2 & $\cdots$ & $R$ \\
\cline { 2 - 5 } Part (V) & 1 & 2 & $\cdots$ & $J$ \\
\cline { 2 - 5 } & & &
\end{tabular}

Figure 3. Scheme of solution representation structure.
I. The first part: $1 \times S$ random vector specifies the priority of the suppliers for transporting materials into the plants;

II. The second part: $1 \times U$ random vector specifies the priority of water resources for transporting water into the plants;

III. The third part: $1 \times I$ random vector specifies the priority of manufacturers for producing the products and transporting them to the DCs;

IV. The fourth part: $1 \times R$ random vector specifies the priority of refineries;

V. The fifth part: $1 \times J$ random vector specifies the priority of DCs for transporting the products to the customers.

Figure 3 represents this structure schematically. In this structure, each gene of vectors is a random number between zero and one. Besides, customers' demands will never exceed the capacity limitations. To clarify the trend of encoding the problem, Figure 4 indicates an example of DCs selection in which $J=5$. In this figure, the random numbers are generated; their positions are kept, and then sorted in an ascending order. Based on our capacity, three of the first genes are selected. The positions of these numbers are selected by DCs (DCs numbers 2, 5, and 1 are selected based on corresponding capacity). Moreover, the continuous decision variables, including $Q_{i p}, Q S_{s i r l}, Q P_{i j p l}, Q D_{j k p l}$, $Q U_{u i l}, Q O_{i r l}$, and $Q R_{\text {ril }}$, are encoded based on upper bounds and randomly generated between zero and their upper bounds. For more details about the structure of this type of representation, one can refer to Hajipour et al. [24].

To prevent the violations of the constraints above, Yeniay and Ankare's method [29] is used to penalize them. Hence, infeasible solutions are found using Eq. (35):

$$
P(x)=M \times\left[\left(\frac{g(x)}{b}\right)-1 \geq 0\right],
$$

where $M, g(x), P(x)$, and $f(x)$ represent a large number, the constraint under consideration, the penalty value, and the objective function value of chromosome $x$, respectively. In this equation that is designed for a constraint such as $g(x) \leq b$, more violations

\begin{tabular}{|c|c|c|c|c|c|}
\hline Generated vector & 0.42 & 0.31 & 0.76 & 0.66 & 0.38 \\
\hline Sorted vector & 0.31 & 0.38 & 0.42 & 0.66 & 0.76 \\
\hline Selected DCs & $(2)$ & (5) & (1) & (4) & (3) \\
\hline
\end{tabular}

Figure 4. An instance of DCs encoding. 
receive bigger penalties. Moreover, the penalty values are considered for all of the three-objective functions through an additive function given in Eq. (36):

$$
F(x)= \begin{cases}f(x) ; & x \in \text { feasible region } \\ f(x)+P(x) ; & x \notin \text { feasible region }\end{cases}
$$

\subsection{VDO main concepts}

VDO is one of the recently known metaheuristic algorithms inspired by the concept of vibration damping in mechanical vibration improvisation of musicians, thus simulating the vibration phenomenon. This algorithm was proposed by Mehdizadeh and TavakkoliMoghaddam [23] and used to solve the parallel machine scheduling problem. The abilities of VDO in the singleobjective problems lead the researchers to use this algorithm in multi-objective cases. Hajipour et al. [24] introduced a multi-objective version of VDO to solve the optimization problems. In this paper, this multiobjective version of VDO algorithm has been developed for discrete environments of the current research.

In an optimization problem using VDO, feasible solutions to the optimization problem can be represented by the status of fluctuation system; rapid quenching can be viewed as local optimization; the optimal solution to the problem can be determined by the minimum energy state.

In the vibration theory, the scope of oscillation, which influences the frequencies of the solutions, plays an important role in defining the loop of algorithm. In other words, high amplitudes lead to a bigger scope of solution and the probability of achieving a new solution is larger in these ranges of amplitudes. Conversely, reducing the amplitudes results in a decrease in the probability of obtaining a new solution, such that the scope of solutions can be reduced by decreasing the range of oscillations, and then the system stops from the amplitude state [23,24].

This vibration damping-based algorithm starts working by initializing random solutions, and the parameters, such as max of iteration at each amplitude $(L)$, initial amplitude $\left(A_{0}\right)$, damping coefficient $(\gamma)$, and standard deviation $(\sigma)$, are initialized. For each solution, an Objective Function Value (OFV) is considered and the evaluation of the solutions can be done based on these OFVs. The algorithm contains the two following main loops:

1. Generating a solution randomly and then obtaining and choosing a new solution based on the neighborhood structure. Regarding the Rayleigh distribution function, a solution with a better OFV is selected. The generated solution is a new one when its OFV is better than previous solution. Otherwise, random number $(r)$ between one and zero is generated, then a new solution is chosen based on the following criteria:

$$
r<1-\exp \left(-\frac{A^{2}}{2 \sigma^{2}}\right) \text {. }
$$

2. Decreasing the amplitude at each iteration as:

$$
A_{t}=A_{0} \exp \left(-\frac{\gamma t}{2}\right) .
$$

Finally, by reaching the stopping criteria, the algorithm is terminated.

\subsection{MOVDO considerations}

To use VDO algorithm in multi-objective functions problems, the comparison of the solutions should be considered with regard to the all objective functions. To do this, Fast Non-Dominated Sorting (FNDS) and Crowding Distance (CD), as the two basic concepts of multi-objective meta-heuristics, are used. In FNDS, initial population $(R)$ is evaluated. In this regard, all solutions are selected using domination theory [30]. By this process, all solutions are divided into different fronts. After sorting the populations based on FNDS, the solutions in the same fronts (equal non-dominated rank) are evaluated based on the $\mathrm{CD}$ measure. The procedure of CD calculation is computed based on [24].

A selection operator should be applied to select individuals of the next generation, so in this paper, a crowded tournament selection operator is used [31]. In this regard, $n$ individuals are chosen randomly. The combination of FNDS and CD measures provides solution's rank in Pareto fronts. The solutions with the least ranks are better and chosen sooner in the new population. Then, in order to assure the elitism, the parents and offspring populations are hybridized, and again, the non-domination sorting is executed until the population size becomes $N$. The MOVDO stops working by reaching predetermined number of iterations.

As shown in Figure 5, the process of the proposed MOVDO starts by generating $P_{j}$ as the initial population of the solution vectors. Then, new population, $Q_{j}$, is created based on applying some operators to $P_{j}$. In order to keep the elitism in the algorithm, $R_{j}$ is obtained by combining $P_{j}$ and $Q_{j}$. Finally, the vectors of $R_{j}$ should be sorted in several fronts based on FNDS and $\mathrm{CD}$. To have a predetermined size, next population $\left(P_{j+1}\right)$ is selected according to the proposed selection method.

Figure 6 illustrates the flowchart of MOVDO algorithm based on the basic operators of a VDO algorithm and the described multi-objective operators. The main multi-objective parts are shown using a different color.

To evaluate the results of the proposed MOVDO algorithm, another prevalent multi-objective metaheuristic algorithm, called NSGA-II, is used to solve 


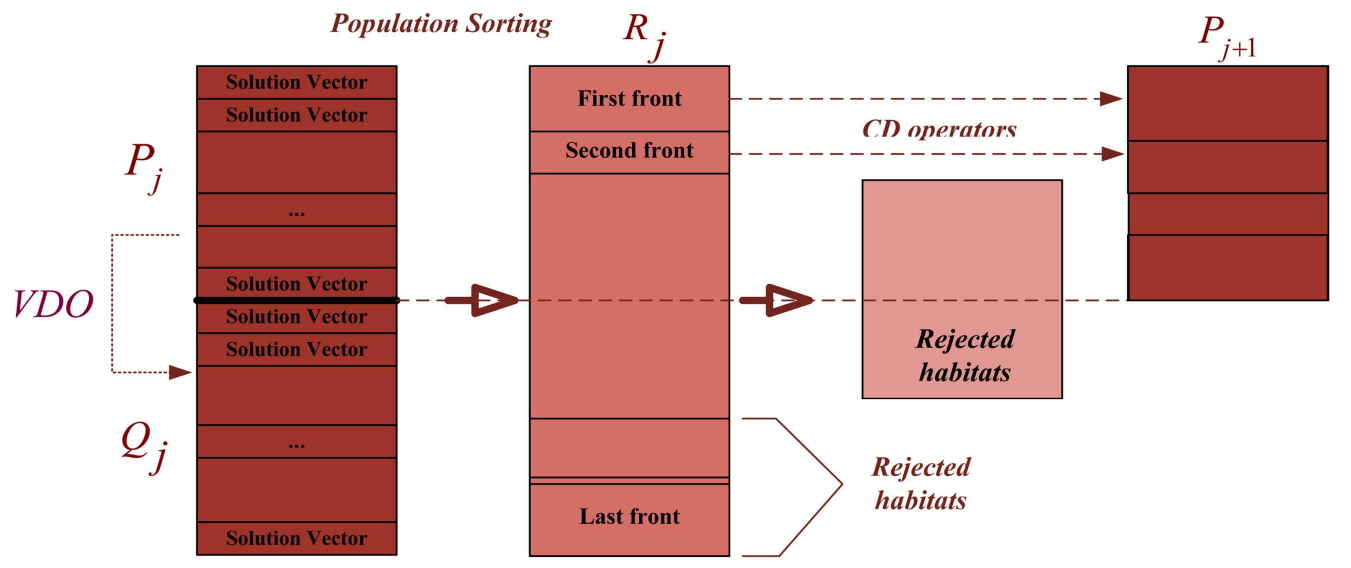

Figure 5. Evolution process of the proposed MOVDO [27].

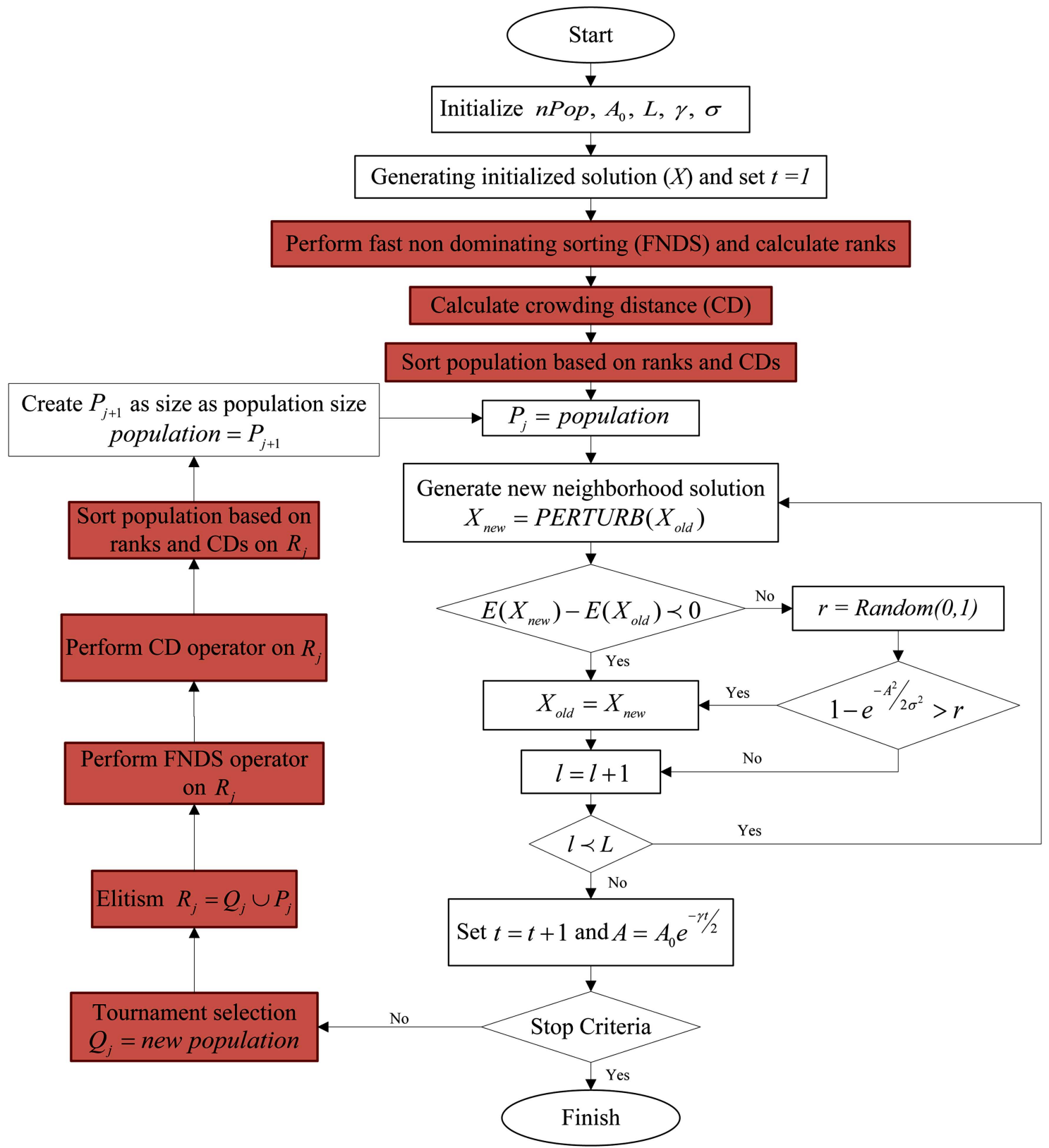

Figure 6. Flowchart of MOVDO [27]. 
the proposed mathematical model. NSGA-II is a modified version of NSGA, which was presented by Srinivas and Deb [32]. To overcome the disadvantages of NSGA, such as the lack of elitism and the complexity of calculations, Deb et al. [31] proposed NSGA-II as a Pareto-based algorithm in which both fast nondominating sorting and CD concepts are considered.

In order to apply the results of both algorithms, their parameters are set. In MOVDO, the numbers of population, initial amplitude, max of iteration at each amplitude, standard deviation, and damping coefficient are 8, 5, 50, 1.5, and 0.04, respectively. In NSGA-II, the numbers of population, crossover probability, mutation probability, and the maximum number of iteration are $20,0.7,0.2$, and 100, respectively.

\section{A case study in textile industry}

In order to demonstrate the applicability of the presented model in real-life situation, a case study was executed in textile industry in Zanjan, Iran. This supply chain consists of suppliers, plants, DCs, WRCs, and customer echelons. Figure 7 represents the region of suppliers, customers, DCs, and water refineries in order to locate them. Planning period is a long-term one for strategic decisions. According to geographical region in this case, a sustainable supply chain is designed to satisfy the demands for two products. The sustainability features in this case are as follows:

- From an environmental viewpoint, two main factors, including water extraction quantity from groundwater and negative effect of industrial wastewater, have been considered;

- From a social viewpoint, providing justice-oriented employment in the studied region has been considered;

- From an economical viewpoint, minimizing the total cost of chain has been applied.

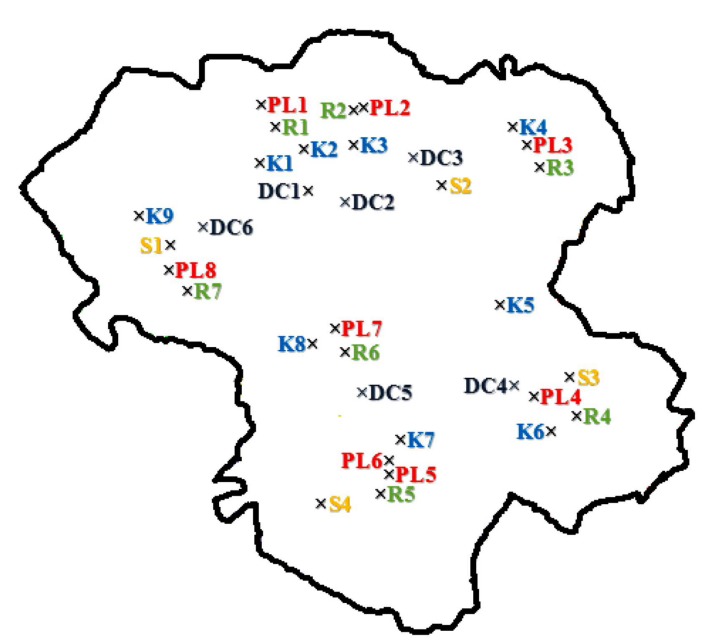

Figure 7. The area of candidate location in supply chain.
To analyze the case, we first give the configuration of this industry in the following subsections. Then, the Pareto optimal solutions are determined by the presented MOVDO.

\subsection{Sets information}

In this case, we have one route between the nodes. All sets of this case study are as follows:

$$
\begin{array}{ll}
s=\left\{S_{i} \mid i=1,2,3,4\right\}, & u=\left\{U_{i} \mid i=1,2, \cdots 7\right\}, \\
r=\left\{R_{i} \mid i=1,2, \cdots 7\right\}, & j=\left\{\mathrm{DC}_{i} \mid i=1,2, \cdots 6\right\}, \\
i=\left\{P L_{i} \mid i=1,2, \cdots 8\right\}, & p=\left\{P_{1}, P_{2}\right\}, \\
m=\left\{M_{1}, M_{2}\right\}, & k=\left\{K_{i} \mid i=1,2, \cdots 9\right\} .
\end{array}
$$

In order to formulate the presented mathematical model, the investigated region is first clustered based on the social and environmental factors. To do so, K-Nearest Neighbor (KNN) clustering approach is applied. As for the environmental factor, water extraction quantity from groundwater and negative effect of industrial wastewater have been considered. As for the social factor, providing justice-oriented employment has been considered. Figures 8-10 indicate the outputs of KNN approach. defined:

According to Figures 8-10, the following sets are

$$
\begin{aligned}
& z=\left\{z_{1}, z_{2}, z_{3}\right\}, \quad w=\left\{w_{1}, w_{2}, w_{3}\right\}, \\
& h=\left\{h_{1}, h_{2}, h_{3}\right\}, \quad D_{w_{2}}=\left\{P L_{3}, R_{3}, U_{3}\right\} \\
& C_{z_{2}}=\left\{P L_{4}, R_{4}, \mathrm{DC}_{4}\right\} \\
& C_{z_{1}}=\left\{\mathrm{DC}_{1}, \mathrm{DC}_{2}, R_{1}, R_{2}, P L_{1}, P L_{2}, \mathrm{DC}_{3}, P L_{3}, R_{3}\right\}, \\
& L_{h_{3}}=\left\{P L_{4}, R_{4}, \mathrm{DC}_{4}, P L_{5}, P L_{6}, R_{5}\right\}, \\
& L_{h_{1}}=\left\{\mathrm{DC}_{1}, \mathrm{DC}_{2}, R_{1}, R_{2}, P L_{1}, P L_{2}\right\} \\
& D_{w_{3}}=\left\{P L_{5}, P L_{6}, R_{5}, \mathrm{DC}_{5}, P L_{7}, R_{6}, U_{5}, U_{6}, U_{7}\right\}, \\
& L_{h_{2}}=\left\{\mathrm{DC}_{3}, P L_{3}, R_{3}, \mathrm{DC}_{5}, P L_{7}, R_{6}, \mathrm{DC}_{6}, P L_{8}, R_{7}\right\}, \\
& C_{z_{3}}=\left\{P L_{5}, P L_{6}, R_{5}, \mathrm{DC}_{5}, P L_{7}, R_{6}, \mathrm{DC}_{6}, P L_{8}, R_{7}\right\}, \\
& D_{w_{1}}=\left\{\mathrm{DC}_{1}, \mathrm{DC}_{2}, R_{1}, R_{2}, P L_{1}, P L_{2}, \mathrm{DC}_{3}, P L_{4},\right. \\
& \left.\quad R_{4}, \mathrm{DC}_{4}, \mathrm{DC}_{6}, P L_{8}, R_{7}, U_{1}, U_{2}, U_{4}, U_{8}\right\} .
\end{aligned}
$$

\subsection{Parameters information}

In this subsection, the collected data for the case in textile industry are provided and reported in Tables 1 to 7 . Other parameters are summarized as in Eqs. (39)(44): 


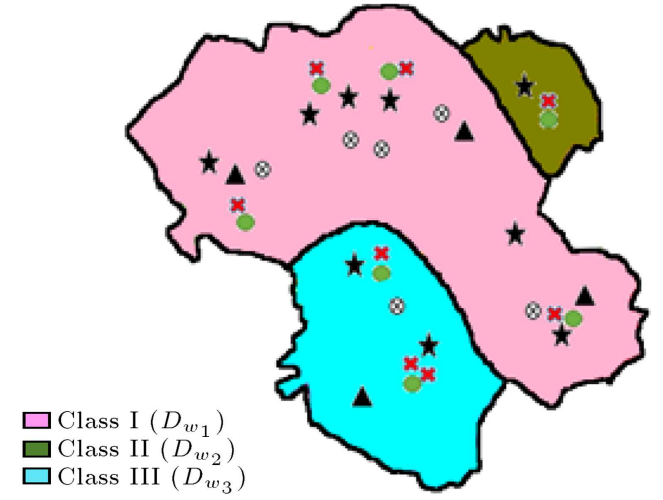

Figure 8. Environmental clustering based on groundwater level.

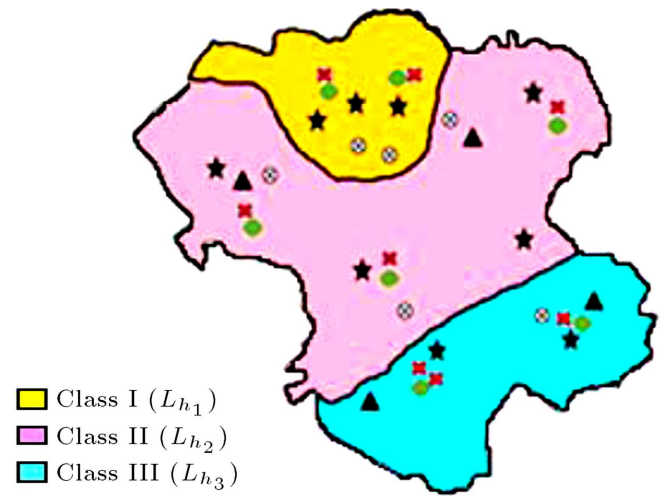

Figure 9. Environmental clustering based on the negative effect of industrial wastewater.

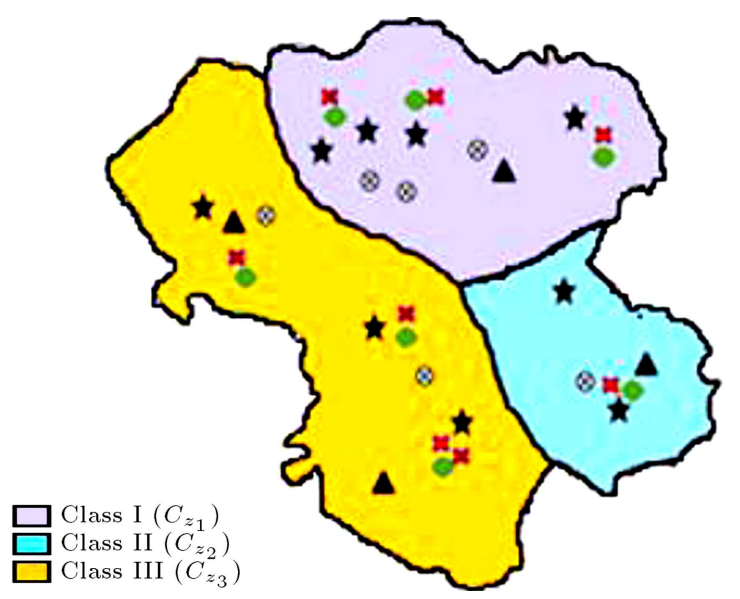

Figure 10. Social clustering based on the need for employment in the regions.

$$
\begin{aligned}
c s_{\text {siml }}= & {\left[\begin{array}{cccccc}
0.06 & 0.065 & 0.07 & 0.08 & 0.07 & 0.07 \\
0.06 & 0.065 & 0.05 & 0.07 & 0.075 & 0.075 \\
0.08 & 0.075 & 0.07 & 0.02 & 0.055 & 0.055 \\
0.08 & 0.08 & 0.08 & 0.06 & 0.04 & 0.035 \\
& 0.065 & 0.04 \\
0.07 & 0.065 \\
0.065 & 0.07 \\
0.05 & 0.065
\end{array}\right]_{S \times I} \quad l \in L S_{s i}, \quad \forall m, \quad(39) }
\end{aligned}
$$$$
c p_{i j p l}=\left[\begin{array}{cccccc}
0.085 & 0.085 & 0.085 & 0.12 & 0.12 & 0.085 \\
0.085 & 0.085 & 0.085 & 0.12 & 0.12 & 0.085 \\
0.1 & 0.2 & 0.08 & 0.11 & 0.11 & 0.12 \\
0.12 & 0.12 & 0.11 & 0.06 & 0.08 & 0.11 \\
0.12 & 0.12 & 0.12 & 0.07 & 0.06 & 0.09 \\
0.12 & 0.12 & 0.12 & 0.07 & 0.06 & 0.09 \\
0.1 & 0.11 & 0.12 & 0.08 & 0.05 & 0.05 \\
0.04 & 0.05 & 0.06 & 0.12 & 0.08 & 0.03
\end{array}\right]_{I \times J}
$$$$
l \in L P_{i j}, \quad \forall p,
$$

$c d_{j k p l}=\left[\begin{array}{ccccc}0.06 & 0.06 & 0.065 & 0.07 & 0.07 \\ 0.06 & 0.06 & 0.06 & 0.07 & 0.07 \\ 0.07 & 0.07 & 0.055 & 0.06 & 0.065 \\ 0.08 & 0.08 & 0.075 & 0.075 & 0.06 \\ 0.07 & 0.07 & 0.08 & 0.09 & 0.05 \\ 0.03 & 0.04 & 0.05 & 0.065 & 0.07\end{array}\right.$

$$
\left.\begin{array}{cccc}
0.08 & 0.08 & 0.075 & 0.065 \\
0.08 & 0.08 & 0.07 & 0.07 \\
0.08 & 0.07 & 0.075 & 0.075 \\
0.02 & 0.06 & 0.07 & 0.08 \\
0.055 & 0.03 & 0.035 & 0.075 \\
0.085 & 0.07 & 0.05 & 0.03
\end{array}\right]_{J \times K}
$$

$$
l \in L D_{j k}, \quad \forall p,
$$

$$
c o_{i r}=\left[\begin{array}{ccccccc}
0.02 & \infty & \infty & \infty & \infty & \infty & \infty \\
\infty & 0.02 & \infty & \infty & \infty & \infty & \infty \\
\infty & \infty & 0.02 & \infty & \infty & \infty & \infty \\
\infty & \infty & \infty & 0.02 & \infty & \infty & \infty \\
\infty & \infty & \infty & \infty & 0.02 & \infty & \infty \\
\infty & \infty & \infty & \infty & 0.025 & \infty & \infty \\
\infty & \infty & \infty & \infty & \infty & 0.02 & \infty \\
\infty & \infty & \infty & \infty & \infty & \infty & 0.02
\end{array}\right],
$$

Table 1. The parameters related to the plant centers.

\begin{tabular}{cccccccccc}
\hline Plant & $\boldsymbol{M} \boldsymbol{P}_{\boldsymbol{i}}$ & $\boldsymbol{F} \boldsymbol{P}_{\boldsymbol{i}}$ & $\boldsymbol{e}_{\boldsymbol{i}}$ & $\boldsymbol{\rho}_{\boldsymbol{i}}$ & $\boldsymbol{\gamma}_{\boldsymbol{i}}$ & $\boldsymbol{\nu}_{\boldsymbol{I} \boldsymbol{P}_{\mathbf{1}}}$ & $\boldsymbol{\nu}_{\boldsymbol{I} \boldsymbol{P}_{\mathbf{2}}}$ & $\boldsymbol{p} \boldsymbol{c}_{\boldsymbol{I} \boldsymbol{P}_{\boldsymbol{1}}}$ & $\boldsymbol{p} \boldsymbol{c}_{\boldsymbol{I} \boldsymbol{P}_{\mathbf{2}}}$ \\
\hline$P L_{1}$ & 380000 & 11000000 & 360 & 0.75 & 5.3 & 2.7 & 4.5 & 17 & 22 \\
$P L_{2}$ & 380000 & 17000000 & 530 & 0.75 & 7.5 & 2.3 & 3.9 & 13 & 19 \\
$P L_{3}$ & 380000 & 11000000 & 360 & 0.75 & 5.3 & 2.7 & 4.5 & 17 & 22 \\
$P L_{4}$ & 380000 & 17000000 & 530 & 0.75 & 7.5 & 2.3 & 3.9 & 13 & 19 \\
$P L_{5}$ & 380000 & 11000000 & 360 & 0.75 & 5.3 & 2.7 & 4.5 & 17 & 22 \\
$P L_{6}$ & 380000 & 11000000 & 360 & 0.75 & 5.3 & 2.7 & 4.5 & 17 & 22 \\
$P L_{7}$ & 380000 & 17000000 & 530 & 0.75 & 7.5 & 2.3 & 3.9 & 13 & 19 \\
$P L_{8}$ & 380000 & 10000000 & 320 & 0.75 & 4.5 & 2.6 & 4.7 & 20 & 27 \\
\hline
\end{tabular}


Table 2. The parameters related to the refinery centers.

\begin{tabular}{cccccc}
\hline $\begin{array}{c}\text { Refinery } \\
\text { center }\end{array}$ & $\boldsymbol{M} \boldsymbol{R}_{\boldsymbol{r}}$ & $\boldsymbol{F} \boldsymbol{R}_{\boldsymbol{r}}$ & $\boldsymbol{h}_{\boldsymbol{r}}$ & $\boldsymbol{\varepsilon}_{\boldsymbol{r}}$ & $\boldsymbol{r} \boldsymbol{c}_{\boldsymbol{r}}$ \\
\hline$R_{1}$ & 6000000 & 1250000 & 33 & 0.63 & 0.013 \\
$R_{2}$ & 6000000 & 1250000 & 28 & 0.53 & 0.015 \\
$R_{3}$ & 8000000 & 1400000 & 23 & 0.47 & 0.011 \\
$R_{4}$ & 6000000 & 1250000 & 43 & 0.53 & 0.015 \\
$R_{5}$ & 9000000 & 1500000 & 68 & 0.47 & 0.011 \\
$R_{6}$ & 6000000 & 1250000 & 43 & 0.53 & 0.015 \\
$R_{7}$ & 6000000 & 1250000 & 43 & 0.53 & 0.015 \\
\hline
\end{tabular}

Table 3. The parameters related to raw materials.

\begin{tabular}{ccccc}
\hline $\begin{array}{c}\text { Raw } \\
\text { material }\end{array}$ & $\boldsymbol{M} \boldsymbol{S}_{\boldsymbol{S}_{\mathbf{1}} \boldsymbol{m}}$ & $\boldsymbol{M} \boldsymbol{S}_{\boldsymbol{S}_{\mathbf{2}} \boldsymbol{m}}$ & $\boldsymbol{M} \boldsymbol{S}_{\boldsymbol{S}_{\mathbf{3}} \boldsymbol{m}}$ & $\boldsymbol{M} \boldsymbol{S}_{\boldsymbol{S}_{\mathbf{4}} \boldsymbol{m}}$ \\
\hline$M_{1}$ & 23000 & 73000 & 70000 & 65000 \\
$M_{2}$ & 83000 & 53000 & 70000 & 68000 \\
\hline
\end{tabular}

Table 4. The parameters related to products.

\begin{tabular}{cccc}
\hline Product & $\boldsymbol{a}_{\boldsymbol{M}_{\mathbf{1}} \boldsymbol{p}}$ & $\boldsymbol{a}_{\boldsymbol{M}_{\mathbf{2}} \boldsymbol{p}}$ & $\boldsymbol{b}_{\boldsymbol{p}}$ \\
\hline$P_{1}$ & 0.6 & 0.4 & 60 \\
$P_{2}$ & 0.5 & 0.5 & 40 \\
\hline
\end{tabular}

Table 5. The parameters related to DCs.

\begin{tabular}{ccccc}
\hline $\mathbf{D C}$ & $\boldsymbol{M} \boldsymbol{D}_{\boldsymbol{j}}$ & $\boldsymbol{F} \boldsymbol{D}_{\boldsymbol{j}}$ & $\boldsymbol{n}_{\boldsymbol{j}}$ & $\boldsymbol{\tau}_{\boldsymbol{j}}$ \\
\hline $\mathrm{DC}_{1}$ & 90000 & 130000 & 37 & 0.7 \\
$\mathrm{DC}_{2}$ & 75000 & 110000 & 33 & 0.5 \\
$\mathrm{DC}_{3}$ & 120000 & 130000 & 37 & 0.7 \\
$\mathrm{DC}_{4}$ & 55000 & 160000 & 47 & 0.8 \\
$\mathrm{DC}_{5}$ & 110000 & 130000 & 37 & 0.7 \\
$\mathrm{DC}_{6}$ & 55000 & 160000 & 47 & 0.8 \\
\hline
\end{tabular}

$c r_{r i}=\left[\begin{array}{cccccc}0.02 & \infty & \infty & \infty & \infty & \infty \\ \infty & 0.02 & \infty & \infty & \infty & \infty \\ \infty & \infty & 0.02 & \infty & \infty & \infty \\ \infty & \infty & \infty & 0.02 & \infty & \infty \\ \infty & \infty & \infty & \infty & 0.02 & 0.025 \\ \infty & \infty & \infty & \infty & \infty & \infty \\ \infty & \infty & \infty & \infty & \infty & \infty\end{array}\right.$

$\left.\begin{array}{cc}\infty & \infty \\ \infty & \infty \\ \infty & \infty \\ \infty & \infty \\ \infty & \infty \\ 0.02 & \infty \\ \infty & 0.02\end{array}\right]$

$c u_{u i}= \begin{cases}0.01 & \text { if } u=i \\ \infty & \text { otherwise }\end{cases}$

$$
\begin{aligned}
& M W_{D_{w_{1}}}=6000000, \\
& M W_{D_{w_{2}}}=6500000, \quad M F_{D_{w_{3}}}=5500000, \\
& \lambda=0.2, \quad \eta_{h}=0.4, \quad \eta_{w}=0.6, \\
& W_{C_{z_{1}}}=0.4, \quad W_{C_{z_{2}}}=0.1, \quad W_{C_{z_{3}}}=0.5, \\
& \psi_{D_{w_{1}}}=0.35, \quad \psi_{D_{w_{2}}}=0.4, \quad \psi_{D_{w_{3}}}=0.25, \\
& M S_{C z_{1}}=300, \quad M S_{C z_{2}}=0, \quad M S_{C z_{3}}=500, \\
& M F_{L_{h_{1}}}=700000, \quad M F_{L_{h_{2}}}=1400000, \\
& M F_{L_{h_{3}}}=1000000 .
\end{aligned}
$$

\subsection{Optimal decisions}

In this section, first, to show the conflict between the objective functions, using the GAMS software, the mathematical model of the problem under study is solved with each objective function separately (in absence of the other two objective functions); the results are presented in Table 8 . The results show that all the three functions are in conflict with each other.

Also, to obtain the Pareto solutions, the problem is solved separately once using the $\varepsilon$-constraint method and another time using the MOVDO algorithm (mentioned in Section 4); the results are presented in Table 9 .

Finally, according to expert viewpoints, Problem 2 (Table 10) (for $\varepsilon$-constraint method) is selected as the ultimate optimal response. The decision variables of this response are as follows:

$$
\begin{array}{lll}
P L_{1}^{*}=1, & P L_{5}^{*}=1, & P L_{6}^{*}=1, \\
\mathrm{DC}_{1}^{*}=1, & \mathrm{DC}_{3}^{*}=1, & \mathrm{DC}_{4}^{*}=1, \\
\mathrm{DC}_{5}^{*}=1, & R C_{1}^{*}=1, & R C_{5}^{*}=1, \\
X_{2111}^{*}=1, & X_{2121}^{*}=1, & X_{4611}^{*}=1, \\
X_{4621}^{*}=1, & X_{3511}^{*}=1, & X_{3521}^{*}=1, \\
T_{111}^{*}=1, & T_{551}^{*}=1, & T_{661}^{*}=1, \\
V_{551}^{*}=1, & V_{651}^{*}=1, & V_{111}^{*}=1, \\
W_{111}^{*}=1, & W_{551}^{*}=1, & W_{561}^{*}=1, \\
Y_{1111}^{*}=1, & Y_{1121}^{*}=1, & Y_{1321}^{*}=1, \\
Y_{5511}^{*}=1, & Y_{5521}^{*}=1, & Y_{5311}^{*}=1, \\
Y_{6311}^{*}=1, & Y_{6321}^{*}=1, & Y_{6321}^{*}=1, \\
Z_{1111}^{*}=1, & Z_{1121}^{*}=1, & Z_{1221}^{*}=1,
\end{array}
$$


Table 6. Water resources capacity.

\begin{tabular}{ccccccccc}
\hline \multirow{2}{*}{ Capacity } & $\boldsymbol{U}_{\mathbf{1}}$ & $\boldsymbol{U}_{\mathbf{2}}$ & $\boldsymbol{U}_{\mathbf{3}}$ & $\boldsymbol{U}_{\mathbf{4}}$ & $\boldsymbol{U}_{\mathbf{5}}$ & $\boldsymbol{U}_{\mathbf{6}}$ & $\boldsymbol{U}_{\mathbf{7}}$ & $\boldsymbol{U}_{\mathbf{8}}$ \\
\cline { 2 - 9 } & 4000000 & 4000000 & 7000000 & 4000000 & 3500000 & 3500000 & 350000 & 4000000 \\
\hline$M U_{U}$ & 40000
\end{tabular}

Table 7. Costumer zones demands.

\begin{tabular}{cccccccccc}
\hline \multirow{2}{*}{ Demand } & \multicolumn{10}{c}{ Costumer zones $(\boldsymbol{k})$} \\
\cline { 2 - 10 } & $\boldsymbol{K}_{\mathbf{1}}$ & $\boldsymbol{K}_{\mathbf{2}}$ & $\boldsymbol{K}_{\mathbf{3}}$ & $\boldsymbol{K}_{\mathbf{4}}$ & $\boldsymbol{K}_{\mathbf{5}}$ & $\boldsymbol{K}_{\boldsymbol{6}}$ & $\boldsymbol{K}_{\boldsymbol{7}}$ & $\boldsymbol{K}_{\mathbf{8}}$ & $\boldsymbol{K}_{\mathbf{9}}$ \\
\hline$D_{K P_{1}}$ & 22000 & 28000 & 24000 & 17000 & 33000 & 13000 & 17000 & 13000 & 17000 \\
$D_{K P_{2}}$ & 15000 & 15000 & 16000 & 12000 & 17000 & 12000 & 12000 & 10000 & 12000 \\
\hline
\end{tabular}

Table 8. Investigation of the conflict between the objective functions of the problem.

\begin{tabular}{cccc}
\hline \multirow{2}{*}{$\begin{array}{c}\text { Objective } \\
\text { function }\end{array}$} & \multicolumn{3}{c}{ The results of solving the model } \\
& \multicolumn{2}{c}{ with different objective functions } \\
\cline { 2 - 4 } & With $\boldsymbol{f}_{\mathbf{1}}(\boldsymbol{x})$ & With $\boldsymbol{f}_{\mathbf{2}}(\boldsymbol{x})$ & With $\boldsymbol{f}_{\mathbf{3}}(\boldsymbol{x})$ \\
\hline$f_{1}(x)$ & $6.05704 \mathrm{E}+07$ & $2.388007 \mathrm{E}+12$ & $2.752911 \mathrm{E}+12$ \\
$f_{2}(x)$ & $2.4476 \mathrm{E}+06$ & $1.93512 \mathrm{E}+06$ & $2.422447391+06$ \\
$f_{3}(x)$ & 595.2 & 845.5 & 1542.4 \\
\hline
\end{tabular}

Table 9. The comparison of the Pareto responses of the $\varepsilon$-constraint and MOVDO methods.

\begin{tabular}{|c|c|c|c|c|c|c|}
\hline \multirow{3}{*}{$\begin{array}{l}\text { Objective } \\
\text { function }\end{array}$} & \multicolumn{3}{|c|}{$\varepsilon$-constraint } & \multicolumn{3}{|c|}{ MOVDO } \\
\hline & \multicolumn{3}{|c|}{ Pareto solution no. } & \multicolumn{3}{|c|}{ Pareto solution no. } \\
\hline & 1 & 2 & 3 & $\mathbf{1}$ & 2 & 3 \\
\hline$f_{1}(x)$ & $8.13052 \mathrm{E}+07$ & $8.10942 \mathrm{E}+07$ & $7.87042 \mathrm{E}+07$ & $8.24148 \mathrm{E}+07$ & $8.33722 \mathrm{E}+07$ & $7.91732 \mathrm{E}+07$ \\
\hline$f_{2}(x)$ & $2.541216 \mathrm{E}+7$ & $2.02634 \mathrm{E}+06$ & $2.74539 \mathrm{E}+06$ & $2.713426 \mathrm{E}+7$ & $2.21523 \mathrm{E}+06$ & $2.86624 \mathrm{E}+06$ \\
\hline$f_{3}(x)$ & 713.4 & 614.2 & 495.2 & 692.5 & 601.4 & 488.2 \\
\hline
\end{tabular}

$$
\begin{aligned}
& Z_{1911}^{*}=1, \quad Z_{1921}^{*}=1, \quad Z_{3211}^{*}=1, \\
& Z_{3311}^{*}=1, \quad Z_{3321}^{*}=1, \quad Z_{3411}^{*}=1, \\
& Z_{3421}^{*}=1, \quad Z_{3521}^{*}=1, \quad Z_{4711}^{*}=1, \\
& Z_{4721}^{*}=1, \quad Z_{5511}^{*}=1, \quad Z_{5811}^{*}=1, \\
& Z_{5821}^{*}=1, \quad Z_{5611}^{*}=1, \quad Z_{5621}^{*}=1, \\
& Q S_{2111}^{*}=53922.2, \quad Q S_{2121}^{*}=46122.2, \\
& Q S_{4611}^{*}=49577.8, \quad Q S_{4621}^{*}=43477.8, \\
& Q S_{3511}^{*}=67400, \quad Q S_{3521}^{*}=50600, \\
& Q U_{111}^{*}=1912710, \quad Q U_{551}^{*}=2560000, \\
& Q U_{661}^{*}=1879290, \quad Q O_{551}^{*}=4800000, \\
& Q O_{651}^{*}=3523667.5, \quad Q O_{111}^{*}=3586332.5, \\
& Q R_{111}^{*}=2869066, \quad Q R_{551}^{*}=3840000, \\
& Q R_{561}^{*}=2818934, \quad Q D_{1111}^{*}=22000, \\
& Q D_{1121}^{*}=15000, \quad Q D_{1221}^{*}=15000,
\end{aligned}
$$

$$
\begin{aligned}
& Q D_{1911}^{*}=17000, \quad Q D_{1921}^{*}=12000, \\
& Q D_{3211}^{*}=28000, \quad Q D_{3311}^{*}=24000, \\
& Q D_{3321}^{*}=16000, \quad Q D_{3411}^{*}=17000, \\
& Q D_{3421}^{*}=12000, \quad Q D_{3521}^{*}=17000, \\
& Q D_{4711}^{*}=17000, \quad Q D_{4721}^{*}=12000, \\
& Q D_{5511}^{*}=33000, \quad Q D_{5811}^{*}=13000, \\
& Q D_{5821}^{*}=10000, \quad Q D_{5611}^{*}=13000, \\
& Q D_{5621}^{*}=12000, \quad Q P_{1111}^{*}=39000, \\
& Q P_{1121}^{*}=42000, \quad Q P_{1321}^{*}=19044.4, \\
& Q P_{5511}^{*}=76000, \quad Q P_{5521}^{*}=34000, \\
& Q P_{5311}^{*}=8000, \quad Q P_{6311}^{*}=61000, \\
& Q P_{6321}^{*}=25955.5, \quad Q P_{6321}^{*}=25955.5, \\
& Q Q_{11}=39000, \quad Q 12=61044.4, \quad Q_{51}=84000, \\
& Q 52=34000, \quad Q 61=61000, \quad Q_{62}=25955.5 .
\end{aligned}
$$


Table 10. Generated test problems.

\begin{tabular}{|c|c|c|c|c|c|c|c|}
\hline $\begin{array}{c}\text { Problem } \\
\text { no. }\end{array}$ & $S$ & $U$ & $\boldsymbol{I}$ & $\boldsymbol{J}$ & $\boldsymbol{K}$ & $\boldsymbol{P}$ & $\boldsymbol{R}$ \\
\hline 1 & 1 & 1 & 1 & 2 & 2 & 1 & 2 \\
\hline 2 & 2 & 1 & 1 & 2 & 2 & 3 & 2 \\
\hline 3 & 2 & 2 & 2 & 2 & 4 & 5 & 5 \\
\hline 4 & 3 & 3 & 3 & 3 & 4 & 10 & 5 \\
\hline 5 & 3 & 5 & 5 & 3 & 10 & 10 & 5 \\
\hline 6 & 3 & 5 & 5 & 3 & 10 & 10 & 10 \\
\hline 7 & 5 & 10 & 10 & 8 & 10 & 25 & 10 \\
\hline 8 & 5 & 10 & 10 & 12 & 20 & 35 & 20 \\
\hline 9 & 5 & 15 & 12 & 15 & 20 & 40 & 20 \\
\hline 10 & 5 & 15 & 12 & 15 & 20 & 45 & 20 \\
\hline 11 & 10 & 15 & 12 & 15 & 20 & 45 & 20 \\
\hline 12 & 10 & 15 & 15 & 15 & 25 & 45 & 20 \\
\hline 13 & 10 & 15 & 15 & 15 & 25 & 50 & 20 \\
\hline 14 & 10 & 20 & 15 & 15 & 25 & 50 & 20 \\
\hline 15 & 10 & 20 & 15 & 15 & 25 & 50 & 20 \\
\hline 16 & 10 & 20 & 15 & 15 & 30 & 50 & 20 \\
\hline 17 & 10 & 20 & 18 & 15 & 30 & 50 & 20 \\
\hline 18 & 10 & 20 & 18 & 15 & 30 & 50 & 20 \\
\hline 19 & 10 & 25 & 20 & 15 & 35 & 55 & 20 \\
\hline 20 & 10 & 25 & 20 & 15 & 35 & 55 & 20 \\
\hline 21 & 12 & 25 & 25 & 15 & 35 & 55 & 20 \\
\hline 22 & 12 & 30 & 30 & 15 & 40 & 60 & 20 \\
\hline 23 & 12 & 30 & 30 & 20 & 40 & 60 & 30 \\
\hline 24 & 12 & 30 & 40 & 20 & 45 & 60 & 30 \\
\hline 25 & 12 & 30 & 40 & 20 & 45 & 80 & 30 \\
\hline 26 & 18 & 50 & 50 & 25 & 50 & 100 & 30 \\
\hline 27 & 18 & 50 & 60 & 25 & 50 & 120 & 40 \\
\hline 28 & 18 & 50 & 60 & 25 & 50 & 140 & 40 \\
\hline 29 & 20 & 60 & 70 & 30 & 60 & 150 & 40 \\
\hline 30 & 20 & 60 & 70 & 30 & 60 & 200 & 40 \\
\hline
\end{tabular}

\section{Result analysis and comparisons}

In this section, the performance comparisons of two meta-heuristic algorithms are investigated. The developed algorithms were coded in MATLAB software Version 7.10.0.499, R2010a [33] environment and the experiments were performed on a two-GHz laptop with eight GB RAM to estimate the response functions. To evaluate and compare the performances of the solution methodologies under different environments, the experiments were implemented on 30 test problems reported in Table 10. Then, these instance problems were solved by MOVDO and NSGA-II.

In order to evaluate the performances of the two multi-objective meta-heuristic algorithms, four metrics [34] are used as follows:

(I) Number Of found Solutions (NOS): Counts the number of the Pareto solutions in Pareto optimal front;

(II) Spacing: Measures the standard deviation of the distances among solutions of the Pareto front;

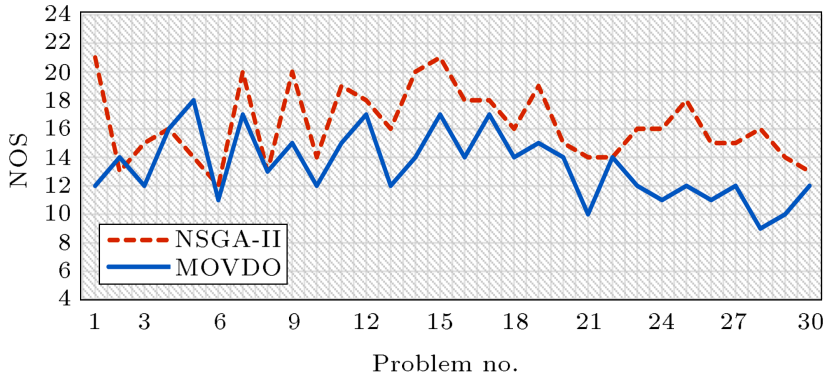

Figure 11. The graphical comparisons of the algorithms in terms of NOS metric.

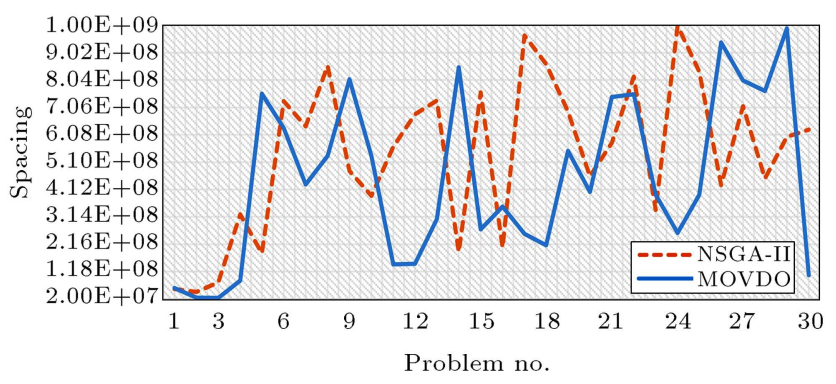

Figure 12. The graphical comparisons of the algorithms in terms of spacing metric.

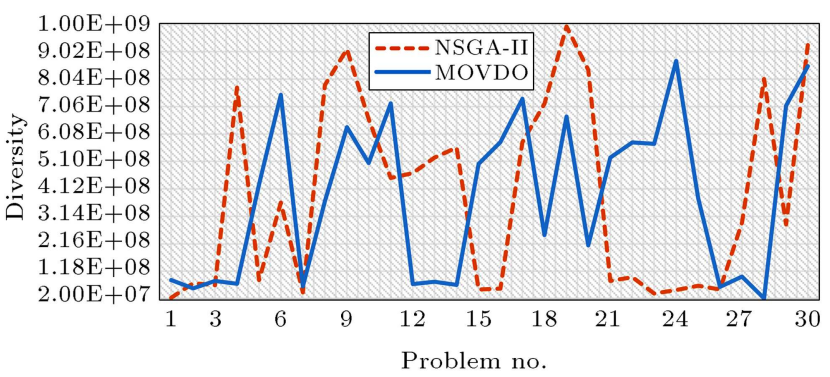

Figure 13. The graphical comparisons of the algorithms in terms of diversity metric.

(III) Diversity: Measures the extension of the Pareto front;

(IV) Computational time of running the algorithms to reach near optimum solutions.

The results of the comparisons in terms of all multi-objective metrics for all algorithms are plotted and compared graphically in Figures 11-14.

As known, in Diversity and NOS metrics, bigger values are desired; for Spacing and $C P U$ time, smaller values prove better. According to the result of the analysis, MOVDO shows better performances in terms of Time. However, in terms of Diversity, Spacing, and NOS metrics, the algorithms almost work similarly. In fact, MOVDO can find and manage Pareto solutions. In order to increase the readability of the proposed MOVDO, Figure 15 represents Pareto solutions obtained by MOVDO. In order to show the convergence objectives, the convergence plots of the three objectives are plotted in Figure 16. 


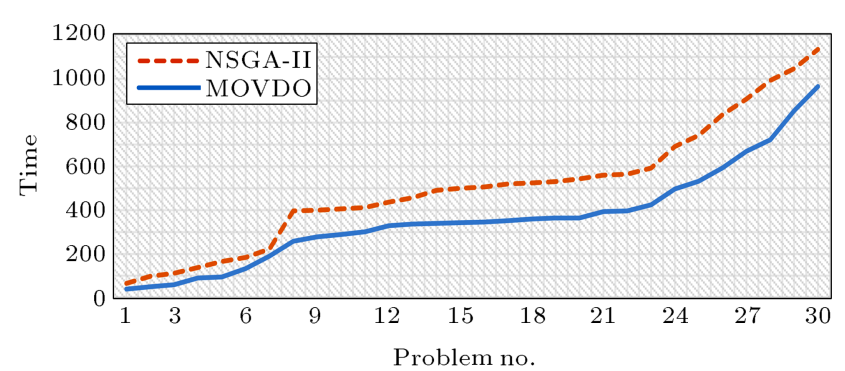

Figure 14. Graphical comparisons of the algorithms in terms of time metric.

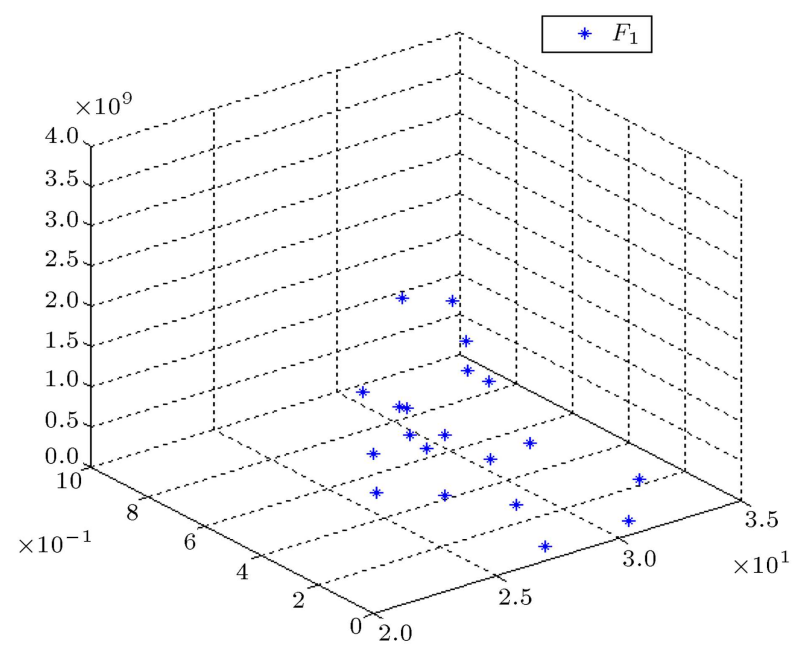

Figure 15. The Pareto solutions for Problem 3

(Table 10) based on the proposed MOVDO.
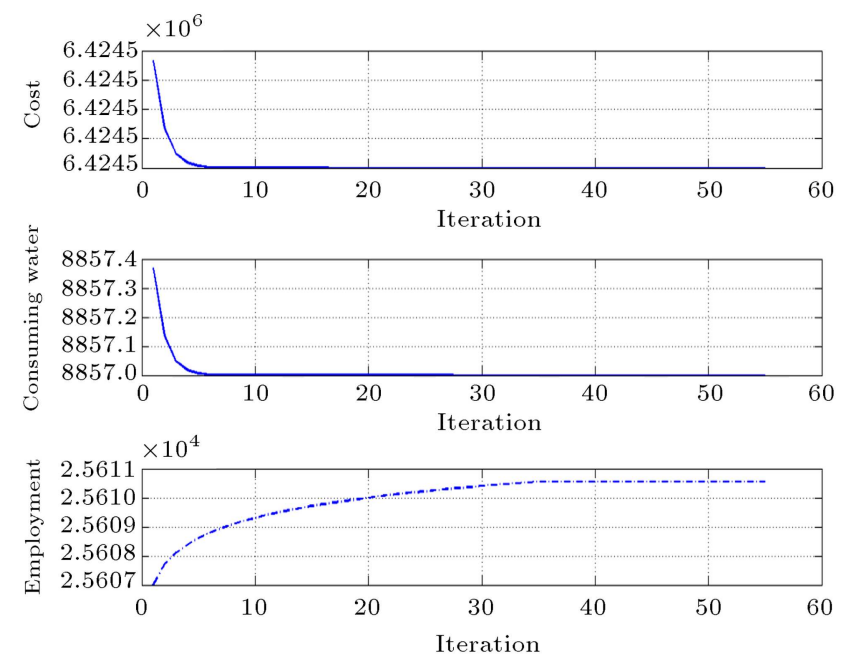

Figure 16. The convergence plots of the three-objective functions for Problem 7 (Table 10) based on the proposed MOVDO.

\section{Conclusion and further research}

In this paper, a multi-objective, multi-echelon and multi-product sustainable supply chain design model was presented in which several suppliers, plants, WRCs, and DCs as different stages of supply chain cooperate with each other to satisfy various costumers' demands. Designing a sustainable closed-loop supply chain concentrating on water recycling is one of the main contributions of this paper. Most research studies have focused on product recycling, while this paper addresses the issue of by-product material recycling in production processes (such as water, oil, and so forth). The objectives were to minimize the total cost of supply chain, minimize the negative environmental effects, and maximize the justice-oriented employment provided by each center simultaneously. Since the proposed mathematical model is NP-Hard, to solve the model, we presented a multi-objective Paretobased meta-heuristic algorithm, called MOVDO, in the supply chain literature. The results of the analysis show the robustness of the proposed MOVDO in terms of computational time. However, in other metrics, MOVDO can compete with the best-developed NSGAII. For future research, one can develop the model in a competitive environment or develop an integrated clustering approach in order to formulate the problem.

\section{References}

1. Simchi-Levi, D., Kaminsky, P. and Simchi-Levi, E., Designing \& Managing the Supply Chain: Concepts, Strategies \& Case Studies, 2nd Ed., New York, NY: McGraw-Hill (2003).

2. Chopra, S. and Meindl, P., Supply Chain Management: Strategy, Planning and Operation, Prentice Hall, Upper Saddle River, USA (2004).

3. Govindan, K., Soleimani, H. and Kannan, D. "Reverse logistics and closed-loop supply chain: A comprehensive review to explore the future", European Journal of Operational Research, 240(3), pp. 603-626 (2015).

4. Seuring, S. and Müller, M. "From a literature review to a conceptual framework for sustainable supply chain management", Journal of Cleaner Production, 16(15), pp. 1699-1710 (2008).

5. Hassanzadeh Amin, S. and Zhang, G. "A multiobjective facility location model for closed-loop supply chain network under uncertain demand and return", Applied Mathematical Modeling, 37, pp. 4165-4176 (2013).

6. Zhang, A., Luo, H.Q. and Huang, G. "A bi-objective model for supply chain design of dispersed manufacturing in China", Int. J. Production Economics, 146, pp. $48-58$ (2013).

7. Tang, C.S. and Zhou, S. "Research advances in environmentally and socially sustainable operations", European Journal of Operational Research, 223(3), pp. 585-594 (2012).

8. Brandenburg, M., Govindan, K., Sarkis, J. and Seuring, S. "Quantitative models for sustainable supply chain management: Developments and directions", European Journal of Operational Research, 233(1), pp. 299-312 (2014). 
9. Wang, F., Lai, X. and Shi, N. "A multi-objective optimization for green supply chain network design", Decision Support Systems, 51(2), pp. 262-269 (2011).

10. Elhedhli, S. and Merrick, R. "Green supply chain network design to reduce carbon emissions", Transportation Research Part D: Transport and Environment, 17(5), pp. 370-379 (2012).

11. Chen, G., Govindan, K. and Golias, M.M. "A queueing network based multi-objective model to reduce truck emissions at container terminals", Transportation Part $E$, 55, pp. 3-22 (2013).

12. Govindan, K., Jafarian, A., Devika, K. and Khodaverdi, R. "Two-echelon multiple-vehicle locationrouting problem with time windows for optimization of sustainable supply chain network of perishable food", International Journal of Production Economics, 152, pp. 9-24 (2014).

13. Kannan, G., Jafarian, A. and Nourbakhsh, V. "Biobjective integrating sustainable order allocation and sustainable supply chain network strategic design with stochastic demand using a novel robust hybrid multiobjective metaheuristic", Computers \& Operations Research, 62, pp. 112-130 (2015).

14. Cakravastia, A., Toha, I. and Nakamura, N. "A twostage model for the design chain networks", International Journal of Production Economics, 80, pp. 231248 (2002).

15. Santoso, T., Ahmed, S., Goetschalckx, M. and Shapiro, A. "A stochastic programming approach for supply chain network design under uncertainty", European Journal of Operational Research, 167, pp. 96-115 (2005).

16. Syam, S.S. "A model and methodologies for the location problem with logistical components", Computers \& Operations Research, 29, pp. 1173-1193 (2002).

17. Syarif, A., Yun, Y. and Gen, M. "Study on multistage logistics chain network: A spanning tree-based genetic algorithm approach", Computers \& Industrial Engineering, 43, pp. 299-314 (2002).

18. Jayaraman, V. and Ross, A. "A simulated annealing methodology to distribution network design and management", European Journal of Operational Research, 144, pp. 629-645 (2003).

19. Yeh, W.C. "A efficient memetic algorithm for multistage supply chain network problem", International Journal of Advance Manufacturing Technology, 29(78), pp. 803-813 (2006).

20. Altiparmak, F., Gen, M., Lin, L. and Paksoy, T. "A genetic algorithm approach for multi-objective optimization of supply chain networks", Computers and Industrial Engineering, 51, pp. 196-215 (2006).
21. Yeh, W.C. "A hybrid heuristic algorithm for multistage supply chain network problem", International Journal of Advance Manufacturing Technology, 26(5$6)$, pp. 675-685 (2005).

22. Moncayo-Martinez, L.A. and Zhang, D.Z. "Multiobjective ant colony optimization: A meta-heuristic approach to supply chain design", International Journal of Production Economics, 131, pp. 407-420 (2011).

23. Mehdizadeh, E. and Tavakkoli-Moghaddam, R. "Vibration damping optimization algorithm for an identical parallel machine scheduling problem", Conference of Iranian Operations Research Society, Babolsar, Iran (May 20-22, 2009).

24. Hajipour, V., Mehdizadeh, E. and TavakkoliMoghaddam, R. "A novel Pareto-based multi-objective vibration damping optimization algorithm to solve multi-objective optimization problems", Scientia Iranica: Transactions E, 21(6), pp. 2368-2378 (2014).

25. Hajipour, V., Khodakarami, V. and Tavana, M. "The redundancy queuing-location-allocation problem: A novel approach", IEEE Transaction of Engineering Management, 61(3), pp. 534-544 (2014b).

26. Hajipour, V., Kheirkhah, A.S., Tavana, M. and Absi, N. "Novel Pareto-based meta-heuristics for solving multi-objective multi-item capacitated lot-sizing problems", International Journal of Advanced Manufacturing Technology, 80, pp. 31-45 (2015).

27. Hajipour, V., Zanjirani Farahani, R. and Fattahi, P. "Bi-objective vibration damping optimization for congested location-pricing problem", Computers \& Operations Research, 70, pp. 87-100 (2016).

28. Hajipour, V., Fattahi, P., Tavana, M. and Di Caprio, D. "Multi-objective multi-layer congested facility location-allocation problem optimization with pareto-based meta-heuristics", Applied Mathematical Modelling, 40, pp. 4948-4969 (2016).

29. Yeniay, O. and Ankare, B. "Penalty function methods for constrained optimization with genetic algorithms", Mathematical and Computational Application, 10, pp. 45-56 (2005).

30. Deb, K., Pratap, A., Agarwal, S. and Meyarivan, T. "A fast and elitist multi-objective genetic algorithm: NSGA-II", IEEE Transactions on Evolutionary Computation, 6, pp. 182-197 (2002).

31. Coello, C.A., Lamont G.B. and Van Veldhuizen, D.A., Evolutionary Algorithms for Solving Multi-Objective Problems, 2nd Ed., Springer, Berlin (2007).

32. Srinivas, N. and Deb, K. "Multi-objective function optimization using nondominated sorting genetic algorithms", Evolutionary Computation, 2(3), pp. 221-248 (1995). 
33. MATLAB Version 7.10.0.499 (R2010a), The MathWorks, Inc. Protected by U.S. and international patents (2010).

34. Zitzler, E. and Thiele, T. "Multi-objective optimization using evolutionary algorithms - a comparative case study", Conference on Parallel Problem Solving from Nature, Amsterdam, pp. 292-301 (1998).

\section{Biographies}

Hamid Reza Jafari is currently a PhD student at College of Industrial Engineering, Science and Research Branch, Islamic Azad University, Tehran, Iran. He obtained his MSc degree in Industrial Engineering from the Islamic Azad University of Qazvin Branch in Iran (2009) and his BSc degree in Industrial Engineering (2004) from Abhar Islamic Azad University. His research interests include sustainable supply chain, green supply chain design, mathematical modeling problems, and design of fuzzy systems.

Mehdi Seifbarghy received his $\mathrm{PhD}$ degree from Sharif University of Technology in 2005. His research area is on facility location and supply chain management. He had more than 40 journal papers in the valid international and national journals.

Manouchehr Omidvari is an Assistant Professor in Industrial Engineering and Safety Science at the Department of Industrial Engineering, The Qazvin Branch, Islamic Azad University. He is a risk assessment man and safety manager in industry by profession. He has published over 40 research University papers on the board theme of occupational safety and health in refereed academic journals and international and national conference proceedings. His current research interests include risk assessment, disaster management, vulnerability assessment, safety management, productivity, and safety system. 